\title{
Four weeks of electrical stimulation improves glucose tolerance in a sedentary overweight or obese Hispanic population
}

\author{
Michelle J Galvan ${ }^{1}$, Michael J Sanchez ${ }^{1}$, Andrew J McAinch $\mathbb{D}^{2,3}$, Jeffrey D Covington ${ }^{4}$, Jason B Boyle ${ }^{1}$ and \\ Sudip Bajpeyi (1)1
}

\author{
${ }^{1}$ Metabolic, Nutrition, and Exercise Research (MiNER) Laboratory, Department of Kinesiology, University of Texas at El Paso, El Paso, Texas, USA \\ ${ }^{2}$ Institute for Health and Sport (IHES), Victoria University, Melbourne, Victoria, Australia \\ ${ }^{3}$ Australian Institute for Musculoskeletal Science (AIMSS), Victoria University, Melbourne, Victoria, Australia \\ ${ }^{4}$ Department of Pathology, University of Massachusetts Medical School, Worcester, Massachusetts, USA \\ Correspondence should be addressed to S Bajpeyi: sbajpeyi@utep.edu
}

\begin{abstract}
Introduction/purpose: Most US adults (54\%) do not meet the minimum exercise recommendations by the American College of Sports Medicine. Neuromuscular electrical stimulation (NMES) is a novel alternate strategy to induce muscle contraction. However, the effectiveness of NMES to improve insulin sensitivity and energy expenditure is unclear. The purpose of this study was to investigate the effects of 4 weeks of NMES on glucose tolerance in a sedentary overweight or obese population.

Methods: Participants ( $n=10$; age: $36.8 \pm 3.8$ years; BMI $=32 \pm 1.3 \mathrm{~kg} / \mathrm{m}^{2}$ ) were randomized into either control or NMES group. All participants received bilateral quadriceps stimulation (12 sessions; $30 \mathrm{~min} / \mathrm{session}$; three times/week at $50 \mathrm{~Hz}$ and $300 \mu s$ pulse width) altering pulse amplitude to either provide low-intensity sensory level (control; tingling sensation) or at high-intensity neuromuscular level (NMES; maximum tolerable levels with visible muscle contraction). Glucose tolerance was assessed by a 3-h oral glucose tolerance test (OGTT), and substrate utilization was measured by indirect calorimetry and body composition via dual X-ray absorptiometry at baseline and after 4 weeks of NMES intervention.

Results: Control and NMES groups had comparable fasting blood glucose, glucose tolerance, substrate utilization, and muscle mass at baseline. Four weeks of NMES resulted in a significant improvement in glucose tolerance measured by OGTT, whereas no change was observed in the control group. There was no change in substrate utilization and muscle mass in both control and NMES groups. Conclusion: NMES is a novel and effective strategy to improve glucose tolerance in an at-risk overweight or obese sedentary population.
\end{abstract} Key Words

\section{Introduction}

Exercise is highly effective in improving insulin sensitivity and metabolic health (1). However, the majority of US adults do not meet the recommended physical activity guidelines, which has significantly contributed to a dramatic increase in obesity, insulin resistance, and type
2 diabetes mellitus (T2DM) (2). Adherence is further challenging for those with obesity and T2DM suffering from defects in lipid oxidation capacity, musculoskeletal pain, and peripheral neuropathy that limit their ability to exercise $(3,4,5,6)$. Walking extended beyond the https://ec.bioscientifica.com https://doi.org/10.1530/EC-21-0533 (c) 2022 The authors Published by Bioscientifica Ltd
- NMES

- insulin resistance

- glycemic control 
periods of time may be challenging, uncomfortable, and/ or painful for individuals with severe obesity, arthritis, physical disabilities, and/or T2DM complications (7). Obese individuals face challenges during weight-bearing movements such as jogging or running and are at a greater risk for injury and pain-related intolerance (8). Individuals with insulin resistance and/or T2DM have a lower physical performance threshold, such as energy expenditure and cardiorespiratory fitness, often due to lower mitochondrial oxidative capacity, presenting a physical burden preventing them from achieving the recommended intensity and duration of exercise (9). Muscle contraction induced by electrical stimulation in human myotubes (in vitro) as well as in isolated rat muscle has been effective to increase glucose uptake in skeletal muscle $(10,11)$. Therefore, the possibility of improving insulin sensitivity by inducing muscle contraction as an alternative therapeutic approach has been of particular interest for populations that are physically inactive and/or are insulin resistant.

Neuromuscular electrical stimulation (NMES) is an alternative strategy to induce involuntary contraction of skeletal muscle via depolarization of the motor axons and nerves being stimulated through an electrical current (12). NMES is a practical, non-invasive, cost-effective, and innovative method to promote an alternative mode of muscle contraction among individuals who are less likely or unable to engage in conventional physical activity. NMES is used frequently in clinical settings utilizing the application of electrical pulses as a mimetic of voluntary contractions for improving neuromuscular function and strength in disused/immobilized limbs (13). It has been well established that muscle contraction effectively increases glucose uptake via an insulin-independent signaling pathway $(14,15)$. Human studies on the effects of NMES-induced muscle contraction on insulin sensitivity are limited to the population with $\operatorname{T} 2 \mathrm{DM}(16,17,18,19$, $20,21,22,23)$ and spinal cord injury (SCI) $(24,25,26,27)$. Two studies conducted in healthy individuals reported an increased acute glucose disposal rate measured by a hyperinsulinemic-euglycemic clamp $(28,29)$. Although the present literature indicates the promising potential of NMES to acutely increase glucose uptake in healthy individuals and to improve insulin sensitivity in a population with T2DM, comprehensive randomized controlled trials to determine the effects of NMES on insulin sensitivity and substrate utilization are limited. Therefore, the primary purpose of this study was to determine the effects of 4 weeks of NMES-induced muscle contractions on glucose tolerance, energy metabolism, and muscle mass in sedentary overweight or obese adults. We hypothesize
4 weeks of NMES will increase glucose tolerance, energy metabolism, and muscle mass.

\section{Materials and methods}

This study, performed at the Metabolic, Nutrition, and Exercise Research (MiNER) Laboratory of the University of Texas at El Paso (UTEP), was approved by the institutional review board of the University of Texas at El Paso. All subjects provided their written informed consent. Subjects were recruited from the US-Mexico border region of El Paso, TX, a region that consists of $82.1 \%$ of individuals who are identifed as being of Hispanic heritage (30). Inclusion criteria for this study were between the ages of 18 and 54, a BMI above $25 \mathrm{~kg} / \mathrm{m}^{2}$, less than 150 min per week of voluntary exercise, and a regular menstrual cycle for premenopausal women. Ten overweight or obese healthy, sedentary Hispanic subjects were enrolled in the study intervention and were randomized in a singleblinded fashion into two groups: control group or NMES group. Subjects with $<60$ min per week of physical activity were determined sedentary via physical activity monitors (ActiGraph Corp., Pensacola, Florida). Subjects were excluded from the study if they were diagnosed with hypertension, cardiovascular disease, or for the evidence of taking anti-hypertensive, lipid-lowering, or insulinsensitizing medications, smoking, excessive alcohol, pregnant, or unwilling to adhere to the study intervention. Participants were assessed for glucose tolerance, substrate utilization, body composition, and strength at baseline following the 4-week intervention (Fig. 1). Subjects had no prior experience with using NMES.

\section{Neuromuscular electrical stimulation protocol}

All participants received NMES intervention at the UTEP MiNER Laboratory under supervision with the QuadStar ${ }^{\circledR}$ II Digital Multi-Modality Combo Device (TENS-INF-NMS) (BioMedical Life Systems, Vista, CA, USA) and eight 5.08 $\mathrm{cm} \times 5.08 \mathrm{~cm}$ square electrodes (BioMedical Life Systems, Vista, CA, USA). Electrodes were placed bilaterally in the proximal location of the quadriceps motor point using anatomical reference points. The stimulation device was set to the cycled biphasic waveform with a pulse duration of $300 \mu$ s and frequency set to $50 \mathrm{~Hz}$ (31). Via alteration in the pulse amplitude, participants assigned to the NMES group received stimulation up to maximum tolerable levels (ensuring protocol adherence) to induce visible muscle contraction, and those assigned to control received

This work is licensed under a Creative Commons Attribution-NonCommercial-NoDerivatives 4.0 enternationad License.ifica com at 04/26/2023 07:41:30AM 


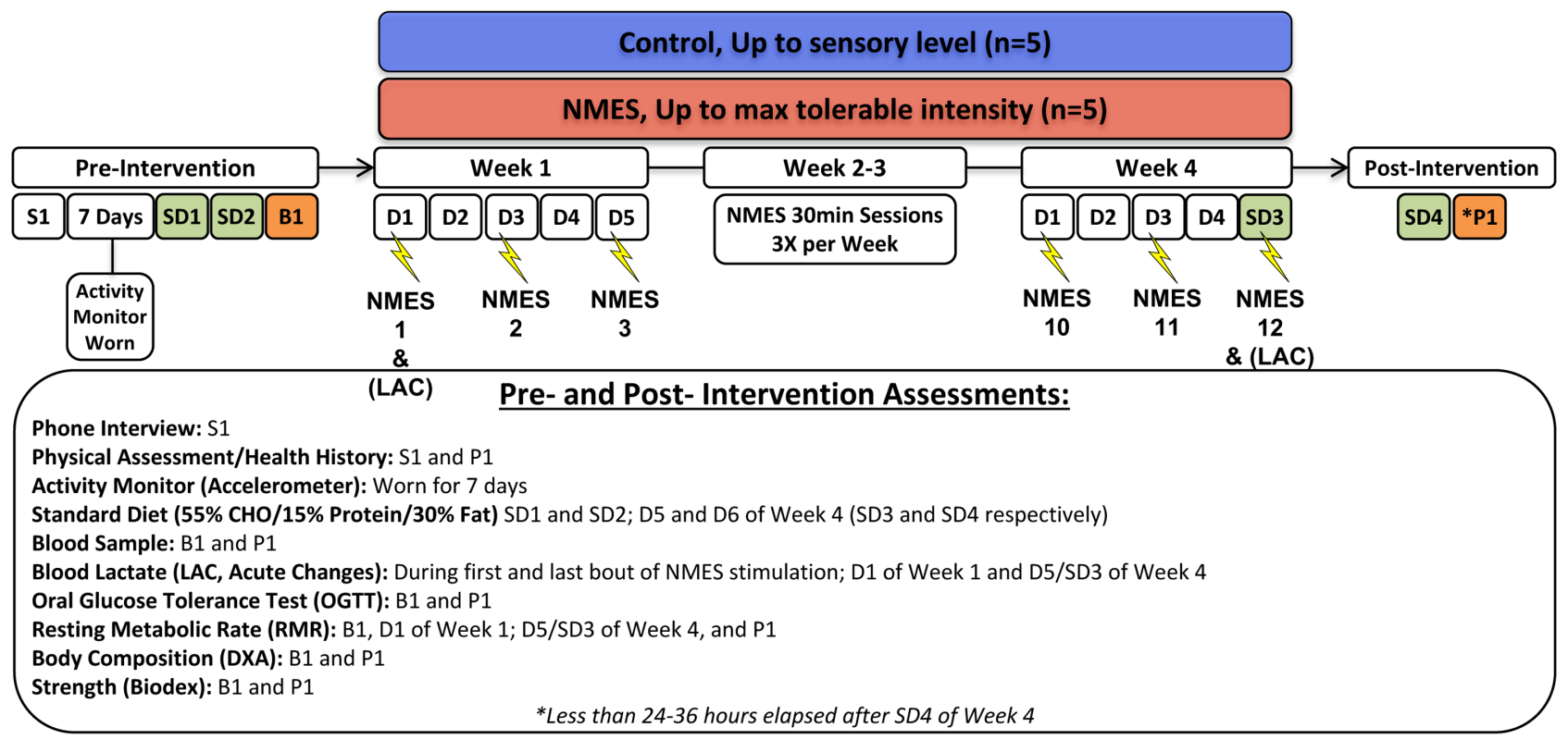

\section{Figure 1}

Study design. B, baseline testing; CHO, carbohydrate; D, day; DXA, dual-energy X-ray absorptiometry; Lac, blood lactate level; NMES, neuromuscular electrical stimulation; P, post-testing; S, screening; SD, standard diet. *Less than 24-36 h elapsed after D5 (SD4) of week 4

stimulation on the lowest possible setting (sensory level: described previously as a tingling sensation). It has previously been demonstrated that NMES between 2 and 8 weeks with two to three sessions per week decrease fasting blood glucose when conducted on quadriceps muscle for 20-30 min per session $(16,20,21,23,27,32,33,34)$. Therefore, in the current study, we employed $30 \mathrm{~min}$ of NMES per session three times per week over a 4-week period. All participants sat upright with their upper body supported (hips $90^{\circ}$ ) and their legs elevated (knees $180^{\circ}$ ) during the stimulation/session.

\section{Body composition}

Body composition was assessed via dual-energy X-ray absorptiometry (DXA) (GE Medical Systems, Madison, WI, USA) during the fasted state. Measurements of total lean mass, total fat mass, bone mineral density, percent body fat, percent android fat, percent gynoid fat, legs percent fat, legs percent lean, leg fat mass/total fat mass ratio, and visceral adipose tissue volume and mass were obtained. Anthropometric measurements of height $(\mathrm{cm})$ via Seca Telescopic Height Measuring Rod and weight (kg) via Tanita WB/11A Class 3 digital scale were obtained to determine and verify a BMI $\left(\mathrm{kg} / \mathrm{m}^{2}\right)$ classified as overweight $\left(25-29.9 \mathrm{~kg} / \mathrm{m}^{2}\right)$ or obese $\left(\geq 30 \mathrm{~kg} / \mathrm{m}^{2}\right)$. Furthermore, the circumference measures of the hips, waist, and mid-thigh were obtained as previously described (2).

\section{Strength}

An Isokinetic Dynamometer Biodex System 3 Pro (Shirley, NY, USA) was used to measure lower limb strength following the standard laboratory protocol. In brief, the participant was seated in the chair, stabilized with cross-body shoulder straps, a waist strap, and thigh straps. The participant's knee was aligned appropriately with the dynamometer shaft and secured to the knee attachment proximal to the medial malleoli. The participant was instructed to fully extend/contract their leg to set the maximum range of motion in both directions. Furthermore, participants were instructed to fully extend their legs again and the knee attachment was locked into place to weigh the leg. The participant was then instructed to perform a series of maximal flexions and extensions of the dominant limb (at $60^{\circ}$ per second).

\section{Dietary control}

Participants were provided with food for 2 days prior to OGTT to control the dietary effects on insulin sensitivity and blood profile. Meals were designed to comply with the USDA 2015-2020 Dietary Guidelines for Americans (35) and individualized to participant preferences/allergies. The standardized diet consisted of macronutrient energy contents of $\sim 55 \%$ carbohydrates, $\sim 15 \%$ protein, and $\sim 30 \%$ fat ( $<10 \%$ of total fat consisting of saturated fat). The Mifflin St. Jeor equation was utilized to match participants to

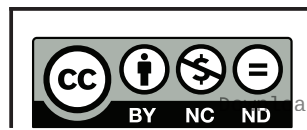

This work is licensed under a Creative Commons Attribution-NonCommercial-NoDerivatives 4.0 International License ifica com at $04 / 26 / 2023$ 07:41:30AM 
their estimated energy requirements (36). Although daily dietary intake was not monitored for the duration of the intervention, participants were encouraged to follow the USDA Dietary Guidelines for Americans (35) and consume an energy-balanced diet.

\section{Glucose tolerance}

Participants were instructed to avoid drinking alcohol, smoking, and strenuous exercise $24-48 \mathrm{~h}$ prior to test days. Following a (12-h) overnight fast, participants arrived at the UTEP Health Sciences Building and were instructed to lie down for 5 minutes prior to obtaining the fasting blood glucose sample. The participant was then asked to orally ingest a drink containing $75 \mathrm{~g}$ of glucose as quickly as possible. Blood samples were then collected at timed intervals of 15, 30, 60, 90, 120, and 180 min following glucose ingestion using CONTOUR ${ }^{\circledast}$ NEXT One, Ascensia Diabetes Care hand-held glucose monitoring system (Parsippany, NJ, USA). Glucose tolerance was assessed by calculating glucose area under the curve (AUC) over the 3-h test. The trapezoid method was used to calculate AUC.

\section{Substrate utilization}

Resting metabolic rate (RMR) and respiratory quotient (RQ) were measured via indirect calorimetry using a Parvomedics TrueOne 2400 metabolic measurement cart (Salt Lake City, UT, USA). On the same day as the OGTT, participants' RMR and RQ were determined by indirect calorimetry prior to glucose ingestion. The acute effect was obtained during the first and last sessions of NMES. RMR and RQ were measured during the $30 \mathrm{~min}$ of NMES application. Energy expenditure was measured using oxygen consumption $\left(\dot{\mathrm{VO}}_{2}\right)$ and carbon dioxide exhalation via indirect calorimetry using Parvomedics TrueOne 2400 metabolic measurement care (Salt Lake City, UT, USA).

\section{Blood lactate}

Blood lactate level was measured during the first and last NMES intervention. Blood lactate was measured by whole blood samples using a hand-held Lactate Plus blood lactate meter (Nova Biomedical, Waltham, MA, USA). A resting/ fasted ( 3-h fast) blood sample was obtained using a lancet prick. Samples from a fingertip were collected prior to stimulation, in intervals of $5 \mathrm{~min}$ during the $30 \mathrm{~min}$ of stimulation. Lactate level over the $30 \mathrm{~min}$ of stimulation was assessed by calculating lactate AUC. Data from the first and last NMES sessions were combined to assess the acute effect of NMES on blood lactate level.

\section{Blood assay samples}

On the same day of the OGTT, a fasting blood sample was obtained via antecubital venipuncture for the analysis of complete blood count (CBC) with differential and platelet count, complete metabolic panel, thyroid profile, plasma lipids, and plasma insulin. The blood samples were evaluated by the Laboratory Corporation of America (Burlington, NC, USA), using their standardized protocols.

\section{Statistical analysis}

Statistical analyses were conducted using GraphPad Prism, version 7.0 (GraphPad Software). Two-way ANOVA with repeated measures and Sidak post hoc analysis were used to compare groups (control and NMES), time (before and after), and group by time effects. For all comparisons, a $P<0.05$ was considered significant, and values are presented as means \pm S.E.M.

\section{Results}

Tables 1 and 2 summarize the participant's baseline characteristics and outcomes following 4 weeks of NMES. Overweight and obese participants $(n=10$; age: $36.8 \pm 3.8$ years; $\mathrm{BMI}=32 \pm 1.3 \mathrm{~kg} / \mathrm{m}^{2}$; body fat: $43.4 \pm 1.7 \%$; waist-tohip ratio (WHR): $0.85 \pm 0.12$ ) were randomized into either control or NMES group. At baseline and post-testing, age, blood pressure, body composition, fasting glucose, lipid profile, thyroid hormones, C-peptide, substrate utilization, and strength were not significantly different between the control and NMES groups. There were no significant changes in CBC parameters, except for a slight decrease in white blood cells after NMES intervention; however, all CBC parameters at baseline and post-testing were within the normal range.

\section{Improvement in glucose tolerance after 4 weeks of NMES}

Fasting blood glucose and glucose levels during an OGTT were not different between groups at baseline as were glucose AUC and C-peptide. While no change in fasting glucose levels was observed (Fig. 2A), there was a significant decrease in glucose AUC $(P=0.0014$; Fig. $2 \mathrm{~B})$ following 4 weeks of NMES. There was no change in C-peptide in the control and NMES groups following 4 weeks of NMES.

This work is licensed under a Creative Commons Attribution-NonCommercial-NoDerivatives 4.0 International License if $i c a$, com at $04 / 26 / 202307: 41: 304 M$ 
Table 1 Descriptive statistics. Data are presented as mean \pm S.E.M.

\begin{tabular}{|c|c|c|c|c|c|c|c|}
\hline & \multicolumn{3}{|c|}{ Control $(n=5)(\mathrm{M} / \mathrm{F}: 1 / 4)$} & \multicolumn{3}{|c|}{ NMES $(n=5)(M / F: 1 / 4)$} & \multirow{2}{*}{$\begin{array}{c}\text { Interaction } \\
P \text {-value }\end{array}$} \\
\hline & Baseline & Post-intervention & $P$-value & Baseline & Post-intervetion & $P$-value & \\
\hline Age (years) & $42.2 \pm 4.95$ & & & $30.33 \pm 4.49$ & & & \\
\hline \multicolumn{8}{|l|}{ Blood pressure } \\
\hline Systolic (mmHg) & $104 \pm 0$ & $103 \pm 2$ & 0.97 & $112 \pm 6$ & $102 \pm 3$ & 0.1 & 0.18 \\
\hline Diastolic (mmHg) & $69 \pm 2$ & $68 \pm 1$ & 0.96 & $79 \pm 6$ & $68 \pm 4$ & 0.03 & 0.08 \\
\hline \multicolumn{8}{|l|}{ Body composition } \\
\hline Height $(\mathrm{cm})$ & $162 \pm 5$ & & & $163 \pm 2$ & & & \\
\hline Body weight (kg) & $87.18 \pm 5.85$ & $86.66 \pm 6.22$ & 0.65 & $87.22 \pm 7.12$ & $86.76 \pm 7.31$ & 0.71 & 0.94 \\
\hline $\mathrm{BMI}\left(\mathrm{kg} / \mathrm{m}^{2}\right)$ & $32.84 \pm 1.44$ & $32.65 \pm 1.51$ & 0.70 & $32.70 \pm 2.29$ & $32.47 \pm 2.38$ & 0.52 & 0.83 \\
\hline Waist circumference & $101.05 \pm 3.33$ & $98.8 \pm 4.27$ & 0.34 & $96.10 \pm 4.73$ & $95.63 \pm 5.54$ & 0.94 & 0.44 \\
\hline Hip circumference & $114.99 \pm 2.86$ & $112.7 \pm 1.85$ & 0.13 & $115.80 \pm 4.57$ & $115.27 \pm 4.60$ & 0.86 & 0.28 \\
\hline Waist-to-hip ratio & $0.88 \pm 0.01$ & $0.88 \pm 0.03$ & 0.98 & $0.83 \pm 0.02$ & $0.83 \pm 0.02$ & 0.99 & 0.94 \\
\hline Lean mass (kg) & $48.55 \pm 5.54$ & $47.98 \pm 5.56$ & 0.14 & $47.30 \pm 4.29$ & $46.77 \pm 4.24$ & 0.17 & 0.92 \\
\hline Fat mass (kg) & $35.73 \pm 2.18$ & $35.61 \pm 1.99$ & 0.97 & $37.02 \pm 3.54$ & $37.21 \pm 3.93$ & 0.92 & 0.69 \\
\hline Body fat (\%) & $42.94 \pm 3.00$ & $43.2 \pm 2.83$ & 0.78 & $43.82 \pm 1.95$ & $44.12 \pm 2.29$ & 0.72 & 0.94 \\
\hline Android fat (\%) & $51.32 \pm 2.73$ & $52.16 \pm 2.39$ & 0.42 & $48.72 \pm 2.97$ & $50.04 \pm 3.40$ & 0.15 & 0.62 \\
\hline Gynoid fat (\%) & $42.22 \pm 3.10$ & $42.78 \pm 2.94$ & 0.13 & $47.58 \pm 3.01$ & $47.06 \pm 3.11$ & 0.16 & 0.02 \\
\hline Android-to-gynoid fat ratio & $1.23 \pm 0.04$ & $1.23 \pm 0.04$ & 0.96 & $1.04 \pm 0.07$ & $1.07 \pm 0.07$ & 0.09 & 0.18 \\
\hline Lean leg mass (kg) & $16.61 \pm 1.88$ & $16.29 \pm 0.12$ & 0.12 & $16.58 \pm 1.91$ & $16.29 \pm 1.91$ & 0.15 & 0.90 \\
\hline \multicolumn{8}{|l|}{ Complete blood count } \\
\hline White blood cells $\left(\times 10^{9} / \mathrm{L}\right)$ & $6.70 \pm 0.46$ & $6.50 \pm 0.58$ & 0.76 & $6.84 \pm 0.90$ & $5.76 \pm 0.62$ & 0.01 & 0.06 \\
\hline Red blood cells $\left(\times 10^{12} / \mathrm{L}\right)$ & $5.00 \pm 0.15$ & $4.90 \pm 0.20$ & 0.41 & $4.78 \pm 0.19$ & $4.93 \pm 0.21$ & 0.11 & 0.04 \\
\hline Plateletes $\left(\times 10^{9} / \mathrm{L}\right)$ & $298.2 \pm 19.60$ & $299.00 \pm 25.12$ & 0.99 & $323.80 \pm 20.98$ & $315.00 \pm 13.66$ & 0.84 & 0.69 \\
\hline Hemoglobin (g/dL) & $14.08 \pm 0.22$ & $13.68 \pm 0.36$ & 0.15 & $13.54 \pm 0.97$ & $13.82 \pm 1.02$ & 0.36 & 0.04 \\
\hline Hematocrit (\%) & $42.94 \pm 0.74$ & $41.48 \pm 1.10$ & 0.14 & $41.18 \pm 2.28$ & $42.20 \pm 2.47$ & 0.35 & 0.04 \\
\hline \multicolumn{8}{|l|}{ Lipid panel } \\
\hline Total cholesterol (mmol/L) & $188.40 \pm 11.77$ & $199.4 \pm 10.28$ & 0.13 & $183.80 \pm 17.35$ & $191.2 \pm 18.70$ & 0.35 & 0.64 \\
\hline Triglycerides (mmol/L) & $169 \pm 31.72$ & $144.8 \pm 9.86$ & 0.50 & $162.20 \pm 37.08$ & $191.2 \pm 26.05$ & 0.99 & 0.44 \\
\hline $\mathrm{HDL}(\mathrm{mmol} / \mathrm{L})$ & $42.40 \pm 5.09$ & $45.2 \pm 6.74$ & 0.45 & $45.80 \pm 2.33$ & $45.2 \pm 1.01$ & 0.96 & 0.32 \\
\hline VLDL (mmol/L) & $33.80 \pm 6.37$ & $29 \pm 2.04$ & 0.52 & $32.40 \pm 7.44$ & $32.4 \pm 5.22$ & 0.99 & 0.46 \\
\hline $\mathrm{LDL}(\mathrm{mmol} / \mathrm{L})$ & $112.20 \pm 7.92$ & $125.2 \pm 8.03$ & 0.03 & $105.60 \pm 11.28$ & $113.6 \pm 14.07$ & 0.18 & 0.42 \\
\hline \multicolumn{8}{|l|}{ Thyroid profile } \\
\hline Triiodothyronine (ng/dL) & $109.20 \pm 7.10$ & $103.20 \pm 7.62$ & 0.52 & $122.80 \pm 2.92$ & $114.80 \pm 6.60$ & 0.33 & 0.8 \\
\hline Thyroxine $\mu \mathrm{g} / \mathrm{dL}$ & $6.38 \pm 0.47$ & $6.84 \pm 0.67$ & 0.31 & $7.72 \pm 0.32$ & $7.36 \pm 0.36$ & 0.47 & 0.09 \\
\hline $\begin{array}{l}\text { Thyroid- stimulating } \\
\text { hormone }(\mu \mathrm{lU} / \mathrm{mL})\end{array}$ & $3.44 \pm 0.90$ & $2.52 \pm 0.43$ & 0.26 & $3.34 \pm 0.71$ & $3.26 \pm 0.35$ & 0.99 & 0.32 \\
\hline C-peptide (ng/mL) & $4.06 \pm 0.56$ & $3.94 \pm 0.51$ & 0.79 & $3.10 \pm 0.57$ & $3.04 \pm 0.42$ & 0.79 & 0.26 \\
\hline
\end{tabular}

\section{Acute and chronic effects of NMES on energy expenditure and substrate utilization}

Four weeks of NMES (chronic effect) showed no significant difference in resting energy expenditure and resting wholebody substrate utilization measured by RQ (Table 2). The acute effect of stimulation showed no significant change in oxygen consumption ( $\dot{\mathrm{VO}}_{2}$; Fig. 3A) at any specific time point or overall during NMES compared to baseline, or when cumulative oxygen consumption was assessed (Fig. 2B). During the 30 min of NMES, no change in wholebody substrate utilization was observed (data not shown). However, lactate concentration significantly increased during min 5 and min $10(P<0.05)$ and tended to increase during min $15(P=0.05)$ in the NMES group compared to the respective resting lactate level (Fig. 3C). Moreover, lactate concentration after 5 and 15 min of NMES stimulation was also greater compared to the respective time points in the control group $(P<0.05)$ (Fig. 3C). Finally, lactate AUC assessed during 30 min of NMES was significantly greater compared to that of the control group ( $P=0.03$; Fig. 3D). It should be noted that when lactate concentration was assessed only for the first session of NMES, a significant increase in lactate concentration was observed at min 10 compared to baseline $(P=0.04)$, and lactate AUC did not reach statistical significance when compared to control $(P=0.10)$.

\section{No change in body composition but decrease in diastolic blood pressure after 4 weeks of NMES}

Body weight, BMI, waist circumference, hip circumference, WHR, blood pressure, body mass, fat mass, percent body

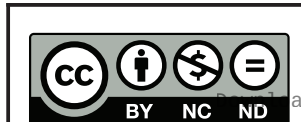

This work is licensed under a Creative Commons Attribution-NonCommercial-NoDerivatives 4.0 Internationad License ifica com at $04 / 26 / 2023 \quad 07: 41: 30 \mathrm{AM}$ 
fat, lean mass, lean leg mass, android percent fat, gynoid percent fat, and android-to-gynoid (A/G) fat ratio were similar between groups at baseline, with no changes observed following 4 weeks of NMES (Table 1). There was a non-significant trend to decrease the systolic blood pressure $(P=0.1)$ and a significant decrease in diastolic pressure $(\mathrm{p}=0.03)$ within group for the NMES group following 4 weeks of intervention (Table 1). There were no changes in blood pressure in the control group. Peak torque per body weight $(\mathrm{TQ} / \mathrm{BW})$ and work to fatigue in both legs were similar between groups at baseline and did not alter following the intervention (Table 2).

\section{No change in lipid profile after 4 weeks of NMES}

Total cholesterol, triglycerides, HDL, VLDL, and LDL showed no difference between the groups at baseline and did not alter following the 4-week intervention in either group (Table 1).

\section{Discussion}

The purpose of this study was to determine the effects of NMES on glucose tolerance, substrate utilization, and muscle mass in a sedentary overweight or obese population. Our data indicate that 4 weeks of NMES resulted in improvement in glucose tolerance, without any effect on resting substrate utilization and muscle mass. Moreover, we demonstrate greater lactate accumulation during acute application of NMES compared to sensory level stimulation (control group). To our knowledge, this is the first randomized comprehensive longitudinal study using NMES in an overweight or obese Hispanic population. This study in the Hispanic population is important as those who are identified as Hispanic have a much greater risk of developing T2DM (37) and thus intervention in this population is required.

Our findings show improvement in glucose tolerance after NMES intervention agreeing with previous studies in populations with T2DM and SCI. An increase in insulin response in patients with T2DM was reported after 2 weeks of NMES treatment (50 Hz of quadriceps stimulation) without any change in lipid profile (21). Furthermore, Catalogna et al. 2016 reported an improvement in blood glucose control in patients with T2DM after daily 5-min stimulation for 2 weeks at $16 \mathrm{~Hz}$ on the anterior aspects of both legs below the kneecap (38). A previous study has shown a greater insulin sensitivity with increasing stimulation intensity (39). Only one study by Wittman et al. 2016 reported no change in fasting blood glucose following a once a week stimulation for 26-week intervention in a sarcopenic obese population (40). The loss of muscle mass that is seen with sarcopenia may explain why no changes were noted in the fasting blood glucose (40). Additionally, there was only one study that reported a decrease in both blood glucose and homeostatic model assessment for insulin resistance following four times a week for 8-week intervention in a population with cystic fibrosis (41).

In our study, NMES treatment significantly increased blood lactate level during the duration of the stimulation, indicating an increase in glucose utilization. Similar results were also reported acutely in a T2DM population in a daily 12-week high-frequency intervention (42). Miyamoto et al. 2012 investigated the acute effect of lower limb NMES for $30 \mathrm{~min}$ at $4 \mathrm{~Hz}, 30 \mathrm{~min}$ after a standard meal in the T2DM population resulting in an increase in $\mathrm{RQ}$, lactate accumulation, and energy expenditure (19). Woelfel et al. 2017 measured the acute effect of NMES on the quadriceps and hamstring muscles over a period of $60 \mathrm{~min}$ at $1 \mathrm{~Hz}$ than at $3 \mathrm{~Hz}$ in the SCI population resulting

Table 2 Resting substrate utilization and strength parameters. Data are presented as mean \pm S.E.M.

\begin{tabular}{l}
\hline Substrate utilization \\
Resting metabolic rate (kcal) \\
Fasting substrate utilization \\
(respiratory quotient) \\
Strength parameters \\
Peak torque per body weight \\
right leg (\%) \\
Peak torque per body weight \\
left leg (\%) \\
Work to fatigue right leg (\%) \\
Work to fatigue left leg (\%)
\end{tabular}

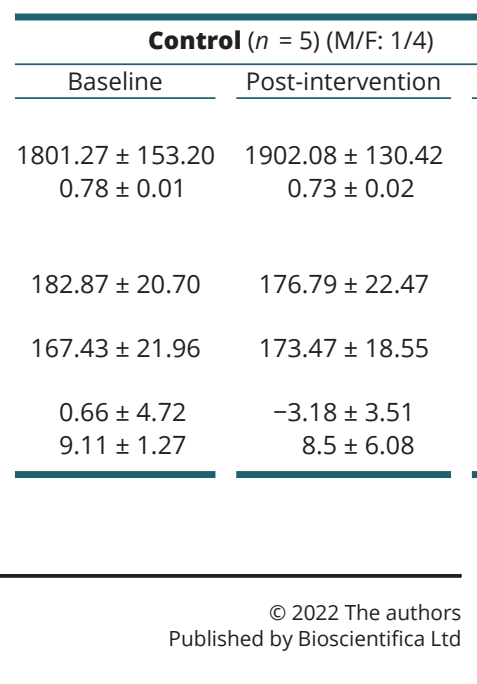

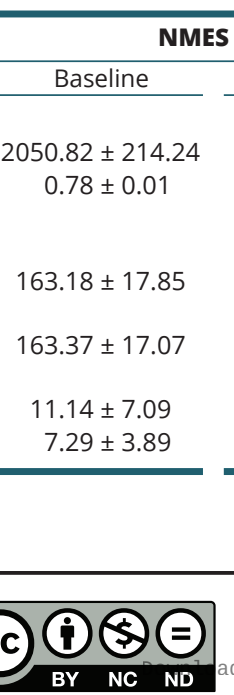

This work is licensed under a Creative Commons Attribution-NonCommercial-NoDerivatives 4.0 Internationab dicense ifica. com at 04/26/2023 07:41:30AM 

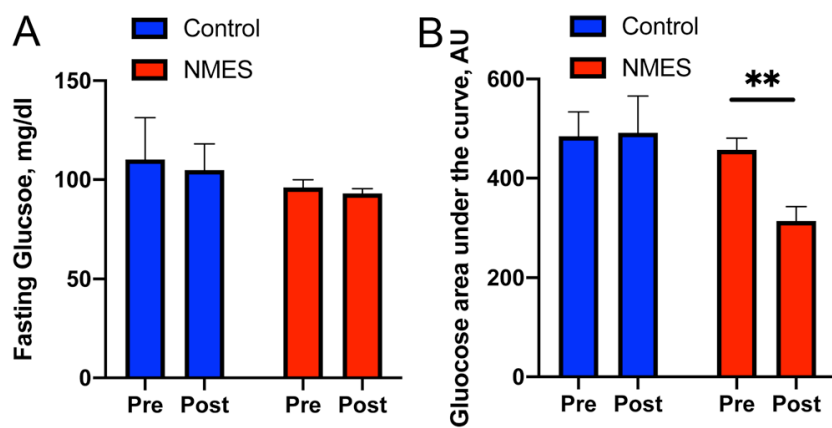

Figure 2

Improvement in glucose tolerance after 4 weeks of NMES, measured by OGTT. Pre- and post-intervention fasting blood glucose (A) and glucose AUC (B) are shown. AU, arbitrary unit; AUC, area under the curve; NMES, neuromuscular electrical stimulation; OGTT, oral glucose tolerance test. *Significant difference in pre- vs post-intervention.

in an increase in energy expenditure (43). However, in the present study, oxygen consumption or energy expenditure showed no changes during NMES stimulation at $50 \mathrm{~Hz}$. The increase in lactate accumulation indicating reliance on glucose utilization is similar to previous studies that investigated the effectiveness of NMES on glycemic control $(19,28,29,44)$. In the present study, lactate AUC was acutely increased in the NMES group. However, our study did not show any effect on whole-body substrate utilization during stimulation, measured by RQ. Given the
RQ represents the whole-body glucose utilization capacity, insulin-sensitive individuals have been shown to be more reliant on whole-body fat oxidation during exercise and are more metabolically flexible (4). It is possible that our study, despite detecting changes in blood lactate levels, was not appropriately powered to detect changes in energy expenditure and whole-body substrate utilization within this small sample size.

Although we observed no improvement in resting energy expenditure and substrate utilization after 4 weeks of NMES, our study is the first, to our knowledge, that evaluated the long-term effect on substrate utilization in an overweight or obese population. Given our study showed a significant increase in lactate AUC in the NMES group compared to the control group, it suggests the role of NMES in muscle contraction-induced glucose utilization, which is in agreement with previous research (45). However, our study showed no significant increase in energy expenditure, contrary to previous research $(19,43,45)$.

Our study shows nochange in body composition and leg muscle mass which is in agreement with previous research using DXA, indicating NMES does not change muscle mass after the intervention; however, it is in disagreement with other research $(17,26)$ in SCI populations after 10 weeks of NMES. Griffin et al. 2008 showed an increase in muscle power and work and a $4 \%$ increase in lean muscle mass by

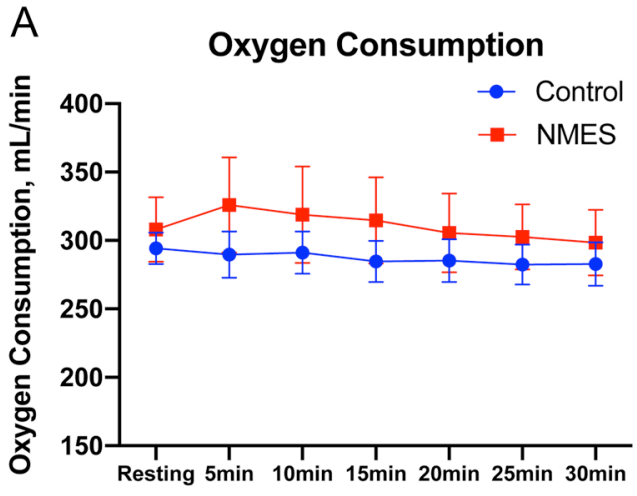

C Lactate revised with legend

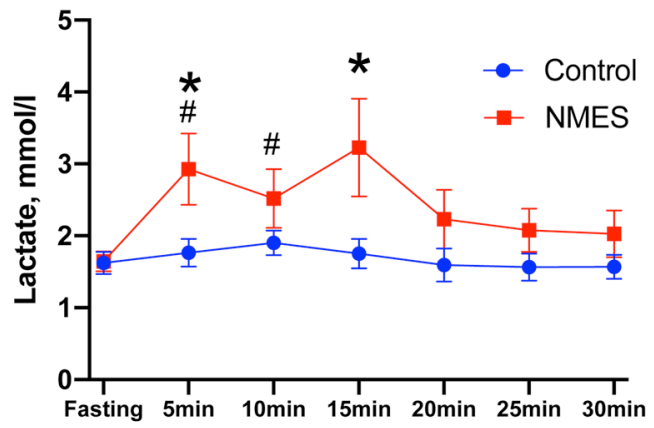

\section{B Oxygen Consumption}

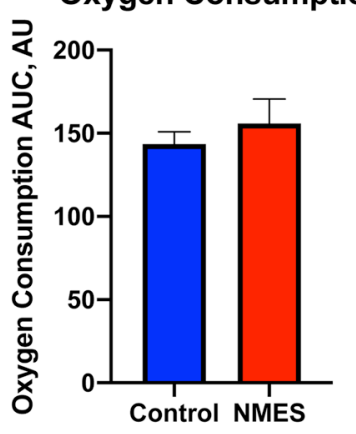

Lactate

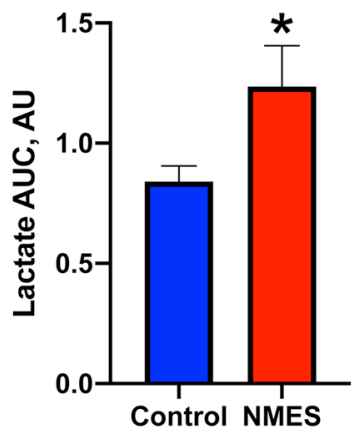

Figure 3

Acute effects of NMES on oxygen consumption (A), oxygen consumption AUC (B), blood lactate level (C), and lactate AUC (D) measured during intervention. $A \cup$, arbitrary unit; $A \cup C$, area under the curve; NMES, neuromuscular electrical stimulation. \#Significantly different compared to baseline. *Significantly different between the control and NMES groups. https://ec.bioscientifica.com https://doi.org/10.1530/EC-21-0533 (c) 2022 The authors Published by Bioscientifica Ltd

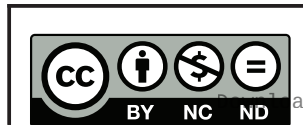

This work is licensed under a Creative Commons Attribution-NonCommercial-NoDerivatives 4.0 Internationab dicense ifica.com at 04/26/2023 07:41:30AM 
DXA using cycling functional electrical stimulation for 10 weeks in a SCI population (26). Two other studies in patients with SCI found an increase in total work output for $30 \mathrm{~min} / \mathrm{session}$, three times per week, for 8 weeks at $30 \mathrm{~Hz}$ (25) and increases in other variables of power, muscle fiber area, and capillarization for two to three times per week for 10 weeks at $50 \mathrm{~Hz}$ (26). Previous studies have shown increases in muscle mass to be accompanied by increases in cross-sectional area and capillary density following NMES interventions (26). The disagreement between our findings and other research could be explained by the differences in neuronal signaling and muscle stimulation following NMES in a SCI population compared to the participants in the current study that was a relatively healthy population without habitual movement impairment.

The primary outcome of our study is an improvement in glucose tolerance in an overweight or obese Hispanic population using a high-stimulation frequency and allowing the participant to use the tolerable intensity to ensure adherence. Our study is the first, to our knowledge, to measure the effects of NMES on android fat, gynoid fat, A/G ratio, and visceral adipose tissue. While, however, the study duration (4 weeks) and/or the stimulation $(50 \mathrm{~Hz})$ may not be adequate to see changes in these measurements or muscle mass, it is possible that there was an improvement in insulin sensitivity indicating possible local effects of muscle contraction-induced signaling pathways to improve glucose uptake.

Our study is limited by the small sample size. However, this study, which is the first randomized control study in a healthy overweight or obese Hispanic population, who are at high risk for developing T2DM, provides compelling evidence for considering NMES as an alternative mechanism to increase insulin sensitivity in this at-risk population. Lack of measurement of insulin is another limitation of our study that does not allow us to directly measure the effects of the intervention on fasting insulin levels or insulin response following the OGTT. Although glucose tolerance has been closely associated with insulin sensitivity, future research is required to confirm our observations of improvement in glucose tolerance measured via OGTT, with the gold standard measurement of insulin sensitivity via a hyperinsulinemic-euglycemic clamp.

In summary, we have demonstrated that 4 weeks of NMES (12 sessions) improves glucose tolerance in a sedentary overweight or obese population. NMES also led to an acute increase in blood lactate concentration during the NMES stimulation. However, this adaption in glucose tolerance occurred without any improvement in resting substrate utilization and muscle mass. Future studies should determine whether the NMES-induced improvement in glucose tolerance offers a novel and effective strategy to improve long-term insulin sensitivity, energy expenditure, and body composition in an at-risk overweight or obese Hispanic population.

\section{Declaration of interest}

The authors declare that there is no conflict of interest that could be perceived as prejudicing the impartiality of the research reported.

\section{Funding}

Applied and Translational Research Funds (PI Bajpeyi), College of Health Sciences, UTEP. Student Research Development Award (SRDA), Texas chapter of American College of Sports Medicine Graduate School Dodson Research Grant, University of Texas at El Paso.

\section{Acknowledgements}

The authors would like to thank all the study participants. In addition, the authors would like to thank Jehu Apaflo, M.S. for his assistance with data analysis, technical assistance, and review of the manuscript, and all members of the MiNER Laboratory. Finally, the authors would like to thank Dr Jeffrey Eggleston for consulting the research team with the strength measurement protocol.

\section{References}

1 American Diabetes Association. New recommendations on physical activity and exercise from people with diabetes. Arlington, VA, USA: American Diabetes Association, 2016. (available at: http:// www.diabetes.org/newsroom/press-releases/2016/ada-issues-newrecommendations-on-physical-activity-and-exercise.html)

2 American College of Sports Medicine. ACSM's Guidelines for Exercise Testing and Prescription, 2014. Baltimore (PA): Lippincott Williams \& Wilkins; 2013.

3 Bajpeyi S, Pasarica M, Moro C, Conley K, Jubrias S, Sereda O, Burk DH, Zhang Z, Gupta A, Kjems L, et al. Skeletal muscle mitochondrial capacity and insulin resistance in type 2 diabetes. Journal of Clinical Endocrinology and Metabolism 201196 1160-1168. (https://doi. org/10.1210/jc.2010-1621)

4 Kelley DE, Goodpaster B, Wing RR \& Simoneau JA. Skeletal muscle fatty acid metabolism in association with insulin resistance, obesity, and weight loss. American Journal of Physiology 1999277 E1130-E1141. (https://doi.org/10.1152/ajpendo.1999.277.6.E1130)

5 Vincent HK, Raiser SN \& Vincent KR. The aging musculoskeletal system and obesity-related considerations with exercise. Ageing Research Reviews 201211 361-373. (https://doi.org/10.1016/j. arr.2012.03.002)

6 Ogilvie RP, Zabetian A, Mokdad AH \& Narayan KMV. Lifestyle characteristics among people with diabetes and prediabetes. In Diabetes in America. Bethesda, MD, USA: National Institute of Diabetes and Digestive and Kidney Diseases (US), 2018. (available at: https:// www.ncbi.nlm.nih.gov/books/NBK567987/)

7 Eves ND \& Plotnikoff RC. Resistance training and type 2 diabetes: considerations for implementation at the population level. Diabetes Care 200629 1933-1941. (https://doi.org/10.2337/dc05-1981) 8 Wanko NS, Brazier CW, Young-Rogers D, Dunbar VG, Boyd B, George CD, Rhee MK, El-Kebbi IM \& Cook CB. Exercise 
preferences and barriers in urban African Americans with type 2 diabetes. Diabetes Educator 200430 502-513. (https://doi. org/10.1177/014572170403000322)

9 Hamasaki H. Daily physical activity and type 2 diabetes: a review. World Journal of Diabetes 20167 243-251. (https://doi.org/10.4239/wjd. v7.i12.243)

10 Nikolic N, Bakke SS, Kase ET, Rudberg I, Halle IF, Rustan AC, Thoresen GH \& Aas V. Electrical pulse stimulation of cultured human skeletal muscle cells as an in vitro model of exercise. PLoS ONE 20127 E33203. (https://doi.org/10.1371/journal.pone.0033203)

11 Santos JM, Benite-Ribeiro SA, Queiroz G \& Duarte JA. The interrelation between aPKC and glucose uptake in the skeletal muscle during contraction and insulin stimulation. Cell Biochemistry and Function 201432 621-624. (https://doi.org/10.1002/cbf.3081)

12 Doucet BM, Lam A \& Griffin L. Neuromuscular electrical stimulation for skeletal muscle function. Yale Journal of Biology and Medicine 2012 85 201-215.

13 Thériault R, Boulay MR, Thériault G \& Simoneau JA. Electrical stimulation-induced changes in performance and fiber type proportion of human knee extensor muscles. European Journal of Applied Physiology and Occupational Physiology 199674 311-317. (https://doi.org/10.1007/BF02226926)

14 Deshmukh A, Coffey VG, Zhong Z, Chibalin AV, Hawley JA \& Zierath JR. Exercise-induced phosphorylation of the novel Akt substrates AS160 and filamin A in human skeletal muscle. Diabetes 200655 1776-1782. (https://doi.org/10.2337/db05-1419)

15 Hayashi T, Hirshman MF, Kurth EJ, Winder WW \& Goodyear LJ. Evidence for 5' AMP-activated protein kinase mediation of the effect of muscle contraction on glucose transport. Diabetes 199847 1369-1373. (https://doi.org/10.2337/diab.47.8.1369)

16 Arsianti RW, Parman DH, Lesmana H \& Taufiqqurohman M. Effect of electrical stimulation in lower extremity as physical exercise in type 2 diabetes mellitus patients. Indonesian Biomedical Journal 201810 62-65. (https://doi.org/10.18585/inabj.v10i1.353)

17 Giggins OM, Crowe L, Coughlan GF \& Caulfield B. Neuromuscular electrical stimulation exercise: a potential alternative to conventional exercise in the management of type 2 diabetes. British Journal of Diabetes 201717 46. (https://doi.org/10.15277/bjd.2017.127)

18 Guzmán-González B, Llanos P, Calatayud J, Maffiuletti NA \& Cruz-Montecinos C. Effect of neuromuscular electrical stimulation frequency on postprandial glycemia, current-related discomfort, and muscle soreness. A crossover study. Applied Physiology, Nutrition, and Metabolism 201944 834-839. (https://doi.org/10.1139/apnm-20180801)

19 Miyamoto T, Fukuda K, Kimura T, Matsubara Y, Tsuda K \& Moritani T. Effect of percutaneous electrical muscle stimulation on postprandial hyperglycemia in type 2 diabetes. Diabetes Research and Clinical Practice 201296 306-312. (https://doi.org/10.1016/j.diabres.2012.01.006)

20 Miyamoto T, Iwakura T, Matsuoka N, Iwamoto M, Takenaka M, Akamatsu Y \& Moritani T. Impact of prolonged neuromuscular electrical stimulation on metabolic profile and cognition-related blood parameters in type 2 diabetes: a randomized controlled crossover trial. Diabetes Research and Clinical Practice 2018142 37-45. (https://doi.org/10.1016/j.diabres.2018.05.032)

21 Sharma D, Shenoy S \& Singh J. Effect of electrical stimulation on blood glucose level and lipid profile of sedentary type 2 diabetic patients. International Journal of Diabetes in Developing Countries 201030194. (https://doi.org/10.4103/0973-3930.70859)

22 van Buuren F, Horstkotte D, Mellwig KP, Fründ A, Vlachojannis M, Bogunovic N, Dimitriadis Z, Vortherms J, Humphrey R \& Niebauer J. Electrical myostimulation (EMS) improves glucose metabolism and oxygen uptake in type 2 diabetes mellitus patients - results from the EMS study. Diabetes Technology and Therapeutics 201517 413-419. (https://doi.org/10.1089/dia.2014.0315)

23 Catalogna M, Doenyas-Barak K, Sagi R, Abu-Hamad R, Nevo U, BenJacob E \& Efrati S. Effect of peripheral electrical stimulation (PES) on nocturnal blood glucose in type 2 diabetes: a randomized crossover pilot study. PLoS ONE 201611 e0168805. (https://doi.org/10.1371/ journal.pone.0168805)

24 Mahoney ET, Bickel CS, Elder C, Black C, Slade JM, Apple Jr D \& Dudley GA. Changes in skeletal muscle size and glucose tolerance with electrically stimulated resistance training in subjects with chronic spinal cord injury. Archives of Physical Medicine and Rehabilitation 2005 86 1502-1504. (https://doi.org/10.1016/j.apmr.2004.12.021)

25 Chilibeck PD, Bell G, Jeon J, Weiss CB, Murdoch G, MacLean I, Ryan E \& Burnham R. Functional electrical stimulation exercise increases GLUT-1 and GLUT-4 in paralyzed skeletal muscle. Metabolism: Clinical and Experimental 199948 1409-1413. (https://doi.org/10.1016/s00260495(99)90151-8)

26 Griffin L, Decker MJ, Hwang JY, Wang B, Kitchen K, Ding Z \& Ivy JL. Functional electrical stimulation cycling improves body composition, metabolic and neural factors in persons with spinal cord injury. Journal of Electromyography and Kinesiology 2009 19 614-622. (https:// doi.org/10.1016/j.jelekin.2008.03.002)

27 Li J, Polston KFL, Eraslan M, Bickel CS, Windham ST, McLain AB, Oster RA, Bamman MM \& Yarar-Fisher C. A high-protein diet or combination exercise training to improve metabolic health in individuals with long-standing spinal cord injury: a pilot randomized study. Physiological Reports 20186 e13813. (https://doi.org/10.14814/ phy2.13813)

28 Hamada T, Hayashi T, Kimura T, Nakao K \& Moritani T. Electrical stimulation of human lower extremities enhances energy consumption, carbohydrate oxidation, and whole body glucose uptake. Journal of Applied Physiology 200496 911-916. (https://doi. org/10.1152/japplphysiol.00664.2003)

29 Hamada T, Sasaki H, Hayashi T, Moritani T \& Nakao K. Enhancement of whole body glucose uptake during and after human skeletal muscle low-frequency electrical stimulation. Journal of Applied Physiology 2003 94 2107-2112. (https://doi.org/10.1152/japplphysiol.00486.2002)

30 Data USA. El Paso, TX. Race and ethnicity. Datawheel, MIT, Deloitte. (available at: https://datausa.io/profile/geo/el-paso-tx/)

31 Sillen MJH, Franssen FME, Gosker HR, Wouters EFM \& Spruit MA. Metabolic and structural changes in lower-limb skeletal muscle following neuromuscular electrical stimulation: a systematic review. PLoS ONE 20138 e69391. (https://doi.org/10.1371/journal.pone.0069391)

32 Arsianti RW, Parman DH \& Lesmana H. Comparison electrical stimulation and passive stretching for blood glucose control type 2 diabetes mellitus patients. In AIP Conference Proceedings. AIP Publishing LLC, 2018.

33 Gorgey AS, Mather KJ \& Gater DR. Central adiposity associations to carbohydrate and lipid metabolism in individuals with complete motor spinal cord injury. Metabolism: Clinical and Experimental 201160 843-851. (https://doi.org/10.1016/j.metabol.2010.08.002)

34 Vivodtzev I, Lacasse Y \& Maltais F. Neuromuscular electrical stimulation of the lower limbs in patients with chronic obstructive pulmonary disease. Journal of Cardiopulmonary Rehabilitation and Prevention 200828 79-91. (https://doi.org/10.1097/01. HCR.0000314201.02053.a3)

35 U.S. Department of Health and Human Services \& U.S. Department of Agriculture. 2015-2020 Dietary Guidelines for Americans, 8th ed. Rockville, MD, USA: Office of Disease Prevention and Health Promotion, 2015. (available at: https://health.gov/our-work/foodnutrition/previous-dietary-guidelines/2015)

36 Mifflin MD, Jeor STSt, Hill LA, Scott BJ, Daugherty SA \& Koh YO. A new predictive equation for resting energy expenditure in healthy individuals. American Journal of Clinical Nutrition 199051 241-247. (https://doi.org/10.1093/ajcn/51.2.241)

37 American Diabetes Association. Statistics about diabetes. Arlington, VA, USA: American Diabetes Association, 2021. (available at: (https:// www.diabetes.org/resources/statistics/statistics-about-diabetes)

38 Catalogna M, Doenyas-Barak K, Sagi R, Abu-Hamad R, Nevo U, BenJacob E \& Efrati S. Effect of peripheral electrical stimulation (PES) on 
nocturnal blood glucose in type 2 diabetes: a randomized crossover pilot study. PLoS ONE 201611 E0168805. (https://doi.org/10.1371/ journal.pone.0168805)

39 Joubert M, Metayer L, Prevost G, Morera J, Rod A, Cailleux A, Parienti JJ \& Reznik Y. Neuromuscular electrostimulation and insulin sensitivity in patients with type 2 diabetes: the ELECTRODIAB pilot study. Acta Diabetologica 201552 285-291. (https://doi.org/10.1007/s00592014-0636-5)

40 Wittmann K, Sieber C, von Stengel S, Kohl M, Freiberger E, Jakob F, Lell M, Engelke K \& Kemmler W. Impact of whole body electromyostimulation on cardiometabolic risk factors in older women with sarcopenic obesity: the randomized controlled FORMOsA-sarcopenic obesity study. Clinical Interventions in Aging 2016 11 1697-1706. (https://doi.org/10.2147/CIA.S116430)

41 Vivodtzev I, Decorte N, Wuyam B, Gonnet N, Durieu I, Levy P, Cracowski JL \& Cracowski C. Benefits of neuromuscular electrical stimulation prior to endurance training in patients with cystic fibrosis and severe pulmonary dysfunction. Chest 2013143 485-493. (https:// doi.org/10.1378/chest.12-0584)
42 Poole RB, Jarrold CP, Burridge JH, Byrne CD \& Holt RIG. Electrical muscle stimulation acutely mimics exercise in neurologically intact individuals but has limited clinical benefits in patients with type 2 diabetes. Diabetes, Obesity and Metabolism 20057 344-351. (https:// doi.org/10.1111/j.1463-1326.2004.00400.x)

43 Woelfel JR, Kimball AL, Yen CL \& Shields RK. Low-force muscle activity regulates energy expenditure after spinal cord injury. Medicine and Science in Sports and Exercise 201749 870-878. (https://doi.org/10.1249/ MSS.0000000000001187)

44 Kimura T, Matsumoto K, Kameda N, Tanaka S, Hayashi T \& Moritani T. Percutaneous electrical muscle stimulation attenuates postprandial hyperglycemia in obese and pre-obese Japanese men. International Journal of Sport and Health Science 20108 1-6. (https://doi.org/10.5432/ ijshs.20090033)

45 Grosset JF, Crowe L, De Vito G, O'shea D \& Caulfield B. Comparative effect of a $1 \mathrm{~h}$ session of electrical muscle stimulation and walking activity on energy expenditure and substrate oxidation in obese subjects. Applied Physiology, Nutrition and Metabolism 201338 57-65. (https://doi.org/10.1139/apnm-2011-0367)

Received in final form 31 December 2021

Accepted 10 January 2022

Accepted Manuscript published online 10 January 2022 (c) 2022 The authors Published by Bioscientifica Ltd
This work is licensed under a Creative Commons Attribution-NonCommercial-NoDerivatives 4.0 Internationad dicense ifica . com at 04/26/2023 07:41:30Am 